\title{
EFEITO DA TEMPERATURA NA VISCOSIDADE APARENTE DE DIFERENTES MARCAS DE REQUEIJÃO CREMOSO TRADICIONAL E LIGHT
}

\section{Temperature effect over the apparent viscosity of conventional and light creamy "Requeijão" from different brands}

\author{
Ana Raisa Paiva ${ }^{1 *}$, Jean Philippe Palma Revillion ${ }^{l}$, Camila Zimmer Dias ${ }^{l}$, \\ Michele Utpott ${ }^{l}$, Plinho Francisco Hertz ${ }^{1}$
}

\begin{abstract}
RESUMO
O Requeijão cremoso é um dos tipos de queijo fundido mais consumidos no Brasil. Ele faz parte de preparações quentes e frias em todo país. Tendo por base o efeito da temperatura na viscosidade de lácteos, o presente trabalho se propôs a avaliar o comportamento reológico de 4 marcas de Requeijão comercial em suas versões tradicional e light, em 3 diferentes temperaturas: 10,25 e $50{ }^{\circ} \mathrm{C}$. Também foi investigada a composição centesimal dos mesmos, no intuito de correlacionar o teor de algum macronutriente e as variações na viscosidade aparente. Os resultados indicam que, em geral, os índices de consistência $(K)$ para requeijões tradicionais foi superior aos seus pares light, excetuando-se as amostras B em $25{ }^{\circ} \mathrm{C}$ e A e C em $50{ }^{\circ} \mathrm{C}$. O efeito da variação da temperatura também foi observado, sendo que invariavelmente um aumento de temperatura resultou em uma diminuição no índice $(K)$ para todas as amostras. O efeito da taxa de cisalhamento $(\dot{\gamma})$ foi acentuado em taxas altas, permitindo que se observasse uma resistência a deformação maior por parte de amostras tradicionais comparadas a amostras light. Nas condições avaliadas, não foi observada correlação entre semelhanças na composição centesimal e curvas de viscosidade aparente.
\end{abstract}

Palavras-chave: consistência; cisalhamento; reologia.

1 Universidade Federal do Rio Grande do Sul, Campus do Vale, Instituto de Ciência e Tecnologia de Alimentos, Avenida Bento Gonçalves, 9500, Prédio 43.212, 91501-970, Porto Alegre, RS, Brasil.

E-mail: ana.paiva@ufrgs.br

* Autor para correspondência. 


\begin{abstract}
Creamy "Requeijão" is one of the most consumed processed cheeses in Brazil. It is used on hot and cold dishes all over the country. Based on the effect of temperature over the apparent viscosity of dairy, this work offered an assessment on the rheological behavior of four commercial Creamy "Requeijão" brands on both traditional and light versions, under 3 different temperatures: 10,25 , and $50^{\circ} \mathrm{C}$. The nutrient composition of the samples was also investigated as to correlate the amount of a certain macronutrient and variations in viscosity. Results show that, in general, consistency indexes $(K)$ of traditional samples were higher than those of their light counterparts, except for sample B at $25^{\circ} \mathrm{C}$ and samples $\mathrm{A}$ and $\mathrm{C}$ at $50{ }^{\circ} \mathrm{C}$. The effect of temperature variations was also observed, and invariably an increase in temperature resulted in a decrease of the $K$ index for all samples. The effect of the shear rate $(\dot{\gamma})$ was accentuated at higher rates, wherein the shear resistance displayed by traditional samples is clearly higher than the resistance displayed by light samples. Under the evaluated conditions, a correlation between nutrient composition and apparent viscosity curves was not observed.
\end{abstract}

Keywords: consistency; shear; rheology.

\section{INTRODUÇÃO}

O Requeijão cremoso é um derivado lácteo tradicionalmente brasileiro, utilizado em preparos quentes e frios, em diversos pratos tradicionais da culinária nacional. Em 2014, o Requeijão e os queijos Mussarela e Prato foram responsáveis por quase $70 \%$ de todo consumo de queijos no Brasil (MILKPOINT, 2015). Entende-se por Requeijão cremoso o produto proveniente da fusão de uma massa coalhada dessorada e lavada, obtida por coagulação ácida e/ou enzimática do leite, com adição de creme de leite e/ou manteiga e/ou gordura anidra de leite e/ou butter oil. A denominação Requeijão aplica-se ao produto cuja base láctea não contenha gordura e/ou proteína de origem não láctea. O Requeijão cremoso deve ter um mínimo de $55 \mathrm{~g} / 100 \mathrm{~g}$ de matéria gorda no extrato seco e um máximo de $65 \mathrm{~g} / 100 \mathrm{~g}$ de umidade (BRASIL, 1997).

Comercialmente, é possível encontrar o Requeijão em versões tradicional e light (ou "leve"): o segundo deve ter uma redução absoluta de pelo menos $3 \mathrm{~g}$ de lipídios por
$100 \mathrm{~g}$ de produto e uma diminuição relativa de pelo menos $25 \%$ da gordura total quando comparado com o produto tradicional de mesma marca (BRASIL, 1998). O apelo saudável de alimentos com teor reduzido de lipídio é fator importante na preferência por produtos light frente a seus similares, visto a existência dessa versão em praticamente todas as marcas dessa categoria de produtos. A demanda por alimentos com teor reduzido de gordura é um desafio para a indústria de alimentos visto a necessidade de manter as características de cremosidade e viscosidade e os atributos sensoriais dos produtos light (RAPACCI, 1997). Em particular, os padrões legais de identidade e qualidade do Requeijão não permitem o uso de espessantes (BRASIL, 1997) e, nesse caso, é a variação na concentração de ingredientes opcionais proteicos que compensaria a diminuição dos teores de gordura nesse produto e incrementaria a sua viscosidade.

Além da composição, fatores como o teor de sólidos, as temperaturas de tratamento térmico do leite, a pressão de homogenei- 
zação e estrutura das micelas (COLLET; TADINI, 2004; PASEEPHOL et al., 2008), influenciam o comportamento reológico de laticínios. Diferentes condições de emulsificação dos ingredientes também afetam a viscosidade. Quando a massa láctea utilizada na formulação dos queijos processados sofre períodos longos de agitação, o tamanho dos glóbulos de gordura é menor, resultando em alimentos mais firmes. A rede proteica dessas massas também auxilia a produção do creme e aumenta a viscosidade do produto final (FU et al., 2017). Contudo, o efeito positivo do incremento da agitação na viscosidade final se limita aos ingredientes utilizados. Quando a amostra em si é submetida a períodos longos ou taxa de cisalhamento superior a $50 \mathrm{~s}^{-1}$ (GONZÁLEZ-TELLO et al., 2009), a viscosidade aparente é mínima e o comportamento de laticínios tende ao newtoniano.

A análise físico-química de amostras comerciais de Requeijão cremoso já foi objeto de pesquisa de diversos autores: Oliveira et al. (2016); Cunha (2007); Garruti et al. (2003). Já as diferenças no comportamento reológico de Requeijão cremoso tradicional e com teor reduzido de gordura foram investigadas anteriormente por Baroni et al. (1999) e Lübeck (2005), sendo que nesses trabalhos foi evidenciado que composição do produto é um parâmetro muito importante na determinação das características reológicas do Requeijão, em particular, a proteína e a gordura contribuem para o aumento da viscosidade e o teor de umidade diminui a pseudoplasticidade do sistema. Contudo, não foi encontrado na literatura pesquisada trabalho que tenha avaliado a viscosidade aparente de marcas comerciais de Requeijão nas versões tradicional e light em diferentes temperaturas, correlacionando os resultados com os teores de umidade, proteína, gordura e carboidrato dos produtos testados.

\section{MATERIAL E MÉTODOS}

\section{Matéria prima}

Amostras de quatro marcas diferentes de Requeijão cremoso comerciais foram adquiridas em um supermercado de Porto Alegre nas versões tradicional (AT, BT, CT e DT) e light (AL, BL, CL e DL). Embora duas das marcas selecionadas sejam gaúchas, uma de Santa Catarina e outra de Minas Gerais, todas são distribuídas nacionalmente e estão amplamente disponíveis em outras redes de supermercado. As amostras foram mantidas em refrigerador $\left(5 \pm 1{ }^{\circ} \mathrm{C}\right)$ e as análises foram efetuadas em até 5 dias após abertura das embalagens.

\section{Composição centesimal}

Cada uma das oito amostras teve o teor de umidade, gordura e proteína analisados em triplicata de acordo com a metodologia da AOAC (1997) e do Instituto Adolfo Lutz (IAL, 2008). O teor de carboidratos e cinzas foi determinado por diferença (\% Carboidratos + cinzas $=100-(\%$ umidade $+\%$ proteína $+\%$ lipídios $)$ ).

\section{Comportamento reológico}

As análises foram realizadas em reômetro rotacional HAAKE MARS III da Thermosystems ${ }^{\circledR}$, cuja temperatura é controlada por placa peltier. A geometria utilizada foi cone-placa, de $60 \mathrm{~mm}$ de diâmetro. Os experimentos foram realizados em 10, 25 e $50{ }^{\circ} \mathrm{C}$. O tempo para cada ensaio foi de 1 minuto em aclimatação na temperatura desejada e 5 minutos, com taxa de cisalhamento constante e crescente (entre 0,01 e $100 \mathrm{~s}^{-1}$ ). Os dados experimentais foram expressos nos modelos da Lei da Potência (eq. 1) e HerschelBulkley (eq. 2). Segundo Santos et al. (2016): 


$$
\begin{aligned}
& \eta=K \dot{\gamma}^{\mathrm{n}-1} \\
& \eta=\tau_{0} / \gamma+K \dot{\gamma}^{\mathrm{n}-1}
\end{aligned}
$$

Onde $\eta=$ viscosidade $($ Pa.s) $K=$ índice de consistência $\left(\mathrm{Pa} . \mathrm{s}^{\mathrm{n}}\right) ; \mathrm{n}=$ índice de comportamento de fluxo (adimensional); $\dot{\gamma}=$ taxa de cisalhamento $\left(\mathrm{s}^{-1}\right) ; \tau_{0}=$ tensão limite de escoamento $(\mathrm{Pa})$. Conforme Eq. 1 , na Lei da Potência a viscosidade é diretamente proporcional aos valores de $K$ e $n$, que são constantes para uma mesma amostra em determinadas condições. A taxa de cisalhamento $\dot{\gamma}$ no teste realizado é crescente e a viscosidade varia de acordo com um incremento na taxa de cisalhamento. Visto que $0<n<1, n$-1 é sempre um valor inferior a zero. Desta forma, o aumento da taxa de cisalhamento é sempre inversamente proporcional à viscosidade do alimento em dado momento.

Já o modelo de Herschel-Bulkley (Eq. 2) leva em consideração a tensão limite de escoamento $\tau_{0}$, que é dividida pela taxa de cisalhamento e então somada à Eq. 1 . Assim como $K$ e n, $\tau_{0}$ é um valor constante. Portanto, $\tau_{0} / \dot{\gamma}$ diminui na medida que a taxa de cisalhamento aumenta. É uma maneira de inserir a influência da tensão de escoamento no cálculo da viscosidade.

\section{RESULTADOS E DISCUSSÃO}

\section{Composição centesimal}

Os valores médios obtidos nas análises de composição centesimal das amostras de Requeijão são apresentados na Tabela 1 . Conforme o requisito legal, todas amostras light apresentam teor de gordura reduzido em pelo menos $25 \%$. Em compensação, das oito amostras analisadas, três amostras light apresentavam teor de umidade acima do permitido pela legislação. Também foi possível demonstrar que todas as amostras de Requeijão cremoso light apresentaram teor de umidade superior a sua versão tradicional e, duas delas apresentavam teores de proteína superiores aos teores de proteína das amostras tradicionais de mesma marca - e a terceira um valor equivalente. Esses resultados são coerentes com aqueles observados por Baroni et al. (1999) e Lübeck (2005). A portaria $\mathrm{n}^{\circ} 359$ do MAPA (BRASIL, 1997) permite a adição de ingredientes opcionais que aumentam o teor de carboidrato e/ou proteína para compensar a redução da gordura, como leite em pó, maltodextrina e concentrado proteico de soro de leite. O intuito é incrementar a rigidez do sistema e manter as características de viscosidade do produto (BARONI et al., 1999).

Tabela 1 - Composição centesimal das amostras de Requeijão cremoso (em g/100g)

\begin{tabular}{cccccc}
\hline Amostras & Umidade & Proteína & Gordura & $\begin{array}{c}\text { Gordura no } \\
\text { extrato seco }\end{array}$ & $\begin{array}{c}\text { Carboidratos } \\
\text { e cinzas }\end{array}$ \\
\hline AT & $61,6 \pm 0,1$ & $8,3 \pm 0,3$ & $28,6 \pm 0,4$ & $74,48 \pm 1,0$ & $1,5 \pm 0,8$ \\
AL & $77,0 \pm 0,2$ & $10,0 \pm 0,2$ & $9,9 \pm 0,1$ & $43,04 \pm 0,4$ & $3,1 \pm 0,5$ \\
BT & $57,0 \pm 0,1$ & $12,0 \pm 0,3$ & $27,1 \pm 0,2$ & $63,02 \pm 0,5$ & $3,9 \pm 0,6$ \\
BL & $66,0 \pm 0,1$ & $15,9 \pm 0,2$ & $11,2 \pm 0,2$ & $32,94 \pm 0,5$ & $6,9 \pm 0,5$ \\
CT & $61,1 \pm 0,1$ & $11,3 \pm 0,3$ & $23,6 \pm 0,2$ & $60,67 \pm 0,5$ & $4,0 \pm 0,6$ \\
CL & $64,4 \pm 0,2$ & $12,9 \pm 0,1$ & $16,7 \pm 0,0$ & $46,91 \pm 0,0$ & $6,0 \pm 0,3$ \\
DT & $61,2 \pm 0,2$ & $13,3 \pm 0,1$ & $21,2 \pm 0,1$ & $54,64 \pm 0,2$ & $4,3 \pm 0,4$ \\
DL & $70,4 \pm 0,1$ & $13,2 \pm 0,2$ & $9,0 \pm 0,2$ & $30,41 \pm 0,6$ & $7,4 \pm 0,5$ \\
\hline
\end{tabular}




\section{Comportamento reológico}

Os resultados obtidos foram descritos de acordo com dois modelos reológicos (Lei da Potência e Herschel-Bulkley) (SÁ et al., 2008). Os parâmetros para ambos modelos estão dispostos na Tabela 2. Ambas equações permitem ajuste satisfatório, porém por Herschel-Bulkley os valores de $\mathrm{R}^{2}$ fo- ram superiores, invariavelmente acima de 0,98 .

Os valores encontrados para $K$ apresentam uma tendência clara de progressão inversamente proporcional à temperatura. Todos os testes realizados confirmam que a medida que a temperatura foi elevada, valores do índice de consistência diminuíram, o que já era esperado para alimentos em geral (SILVA,

Tabela 2 - Parâmetros das equações Lei da Potência e Herschel-Bulkley para as amostras de Requeijão cremoso em temperaturas de 10,25 e $50{ }^{\circ} \mathrm{C}$

\begin{tabular}{|c|c|c|c|c|c|c|c|c|}
\hline \multirow{2}{*}{ Amostra } & \multirow{2}{*}{$\begin{array}{c}\text { Temp. } \\
\left({ }^{\circ} \mathrm{C}\right)\end{array}$} & \multicolumn{4}{|c|}{ Lei da Potência } & \multicolumn{2}{|c|}{ Herschel-Bulkley } & \multirow[b]{2}{*}{$\mathrm{R}^{2}$} \\
\hline & & $K$ & $n$ & $\mathrm{R}^{2}$ & $K$ & $n$ & $\tau_{0}$ & \\
\hline \multirow{3}{*}{ AT } & 10 & 237,00 & 0,025 & 0,9821 & 3455 & 0,0168 & -3279 & 0,9992 \\
\hline & 25 & 58,49 & 0,196 & 0,9683 & 3505 & 0,0080 & -3459 & 0,9912 \\
\hline & 50 & 18,11 & 0,248 & 0,9982 & 201,8 & 0,0305 & $-182,3$ & 0,9994 \\
\hline \multirow{3}{*}{$\mathrm{AL}$} & 10 & 95,28 & 0,155 & 0,9715 & 2129 & 0,0103 & -1990 & 0,9996 \\
\hline & 25 & 41,79 & 0,214 & 0,9870 & 1598 & 0,0090 & -1554 & 0,9994 \\
\hline & 50 & 20,21 & 0,181 & 0,9947 & 500,4 & 0,0129 & $-481,7$ & 0,9992 \\
\hline \multirow{3}{*}{ BT } & 10 & 306,59 & 0,377 & 0,9530 & 463,2 & 0,3322 & $-159,9$ & 0,9950 \\
\hline & 25 & 100,91 & 0,487 & 0,9804 & 122,8 & 0,4577 & $-34,52$ & 0,9964 \\
\hline & 50 & 12,97 & 0,488 & 0,9905 & 19,89 & 0,3807 & $-5,355$ & 0,9928 \\
\hline \multirow{3}{*}{$\mathrm{BL}$} & 10 & 246,03 & 0,299 & 0,9937 & 370,6 & 0,2075 & $-70,5$ & 0,9988 \\
\hline & 25 & 129,35 & 0,213 & 0,9913 & 7795 & 0,0081 & -7715 & 0,9948 \\
\hline & 50 & 6,92 & 0,437 & 0,9917 & 5,788 & 0,4703 & 1,658 & 0,9980 \\
\hline \multirow{3}{*}{$\mathrm{CT}$} & 10 & 103,44 & 0,490 & 0,9704 & 100,1 & 0,4829 & 40,95 & 0,9990 \\
\hline & 25 & 80,23 & 0,326 & 0,9504 & 129,1 & 0,2735 & $-56,98$ & 0,9958 \\
\hline & 50 & 6,09 & 0,602 & 0,9529 & 2,47 & 0,7797 & 10,12 & 0,9898 \\
\hline \multirow{3}{*}{$\mathrm{CL}$} & 10 & 61,71 & 0,423 & 0,9919 & 110,5 & 0,2765 & $-30,38$ & 0,9978 \\
\hline & 25 & 35,23 & 0,387 & 0,9819 & 57,3 & 0,3077 & $-24,19$ & 0,9982 \\
\hline & 50 & 7,60 & 0,444 & 0,9837 & 8,797 & 0,3943 & 0,147 & 0,9964 \\
\hline \multirow{3}{*}{ DT } & 10 & 162,43 & 0,397 & 0,9796 & 290,0 & 0,3494 & $-46,17$ & 0,9848 \\
\hline & 25 & 66,72 & 0,337 & 0,9551 & 255,8 & 0,1490 & $-187,7$ & 0,9851 \\
\hline & 50 & 49,37 & 0,246 & 0,9803 & 176,9 & 0,0335 & $-88,89$ & 0,9944 \\
\hline \multirow{3}{*}{ DL } & 10 & 81,35 & 0,640 & 0,9605 & 89,89 & 0,626 & $-9,486$ & 0,9912 \\
\hline & 25 & 41,72 & 0,591 & 0,9831 & 46,91 & 0,5698 & $-6,779$ & 0,9970 \\
\hline & 50 & 11,74 & 0,508 & 0,9979 & 16,44 & 0,4335 & $-5,184$ & 0,9986 \\
\hline
\end{tabular}


2000) e especificamente para lácteos, com base no descrito por Ramos et al. (2014) e Mathias et al. (2013).

Nas condições propostas, não foi possível identificar correlação entre a composição centesimal e o decréscimo de viscosidade de acordo com a elevação de temperatura. As amostras AL e DL apresentam teores semelhantes de gordura. No entanto, isso não resultou em comportamento reológico semelhante. BT e CT têm quase a mesma quantidade de carboidrato + cinzas e isso também não se traduz em semelhança no comportamento reológico. O teor de umidade semelhante também não se traduz em comportamento reológico análogo, conforme era esperado de acordo com Dimitreli; Thomareis (2008). DT e DL (teores praticamente iguais de proteína) também possuem comportamento diverso. Isso indica que valores isolados de umidade, proteína, gordura e carboidrato + cinzas não são suficientes para prever comportamento de viscosidade em função da temperatura.

Lübeck (2005), identificou que um maior teor de proteína da amostra de Requeijão cremoso resultava em maiores valores para $K$, enquanto maior umidade resultava em menor $K$. Em seus experimentos, as amostras eram preparadas em condições idênticas, mantendo os ingredientes e variando apenas a proporção entre eles. Em amostras comerciais, as diferenças entre as amostras são maiores, visto que há possibilidade de optar por diferentes fornecedores, matérias primas, binômios tempo/temperatura para obtenção da massa, etc. Desta forma, as particularidades das formulações ou condições de processamento (equipamentos de fabricação com diferentes tratamentos mecânicos, térmicos; tempo de aquecimento e tempo de agitação em cada etapa, por exemplo) parecem ser mais relevantes que a composição centesimal.

Dentre amostras da mesma marca, comparando-se formulações light e tradicio- nais, observou-se que, em baixa temperatura $\left(10^{\circ} \mathrm{C}\right)$, todas amostras tradicionais apresentam resultados para $K$ superiores àqueles encontrados para suas respectivas marcas com teor reduzido de gordura. Em temperatura ambiente ou elevada, esse fenômeno apresenta algumas exceções (para ambos ajustes, em $50{ }^{\circ} \mathrm{C}, K_{\mathrm{AL}}>K_{\mathrm{AT}}$ e $K_{\mathrm{CL}}>K_{\mathrm{CT}}$ e em $25^{\circ} \mathrm{C}$ $\left.K_{\mathrm{BL}}>K_{\mathrm{BT}}\right)$. Tais anomalias podem ser resultado de diferentes condições de processamento de requeijões cremosos tradicionais e light dentro de uma mesma marca ou de ingredientes presentes nas formulações. A Tabela 3 é uma transcrição dos dados contidos nos rótulos de cada amostra.

Ambas as amostras que apresentaram $K$ superior para formulações light em $50^{\circ} \mathrm{C}$ $\left(K_{\mathrm{AL}}>K_{\mathrm{AT}}\right.$ e $\left.K_{\mathrm{CL}}>K_{\mathrm{CT}}\right)$ contém concentrado proteico de soro de leite entre os ingredientes dos produtos com teor reduzido de gordura. A marca $A$ apenas na formulação de $A L$ e a marca $\mathrm{C}$ tanto em CT quanto em CL. De acordo com González-Tello et al. (2009), o teor de concentrado proteico de soro de leite tem impacto direto na viscosidade aparente de amostras em temperaturas diferentes. Por exemplo, em taxas de cisalhamento superiores a $50 \mathrm{~s}^{-1}$ (quando o comportamento de soluções de concentrado proteico de soro de leite é newtoniano), amostras contendo $20 \%$ de concentrado a $10{ }^{\circ} \mathrm{C}$ apresentaram viscosidade aparente de $16 \mathrm{mPa}$.s. A $20^{\circ} \mathrm{C}$, esse parâmetro foi aproximadamente $11 \mathrm{mPa}$.s. Já para concentrações de $15 \%$, os resultados foram $8 \mathrm{mPa}$.s a $10{ }^{\circ} \mathrm{C}$ e $5 \mathrm{mPa} . \mathrm{s}$ a $20{ }^{\circ} \mathrm{C}$ e em $10 \%$, os autores obtiveram $4 \mathrm{mPa} . \mathrm{s}$ em $10{ }^{\circ} \mathrm{C}$ e $3 \mathrm{mPa} . \mathrm{s}$ em $20{ }^{\circ} \mathrm{C}$. Desta forma, em baixas temperaturas o efeito da concentração foi facilmente observado. No entanto, o concentrado de proteínas do soro do leite é susceptível à desnaturação proteica. O trabalho de Atuonwu et al. (2017) salientou a relevância de temperaturas elevadas de processamento nas condições de desnaturação, concluindo que amostras que sofreram 
Tabela 3 - Ingredientes de cada amostra de acordo com os respectivos rótulos

\begin{tabular}{|c|c|}
\hline Amostra & Ingredientes \\
\hline $\mathrm{AT}$ & $\begin{array}{l}\text { Creme de leite, massa para produtos lácteos fundidos (leite pasteurizado, fermento } \\
\text { lácteo, cloreto de cálcio e coagulante), leite em pó desnatado, cloreto de sódio, } \\
\text { estabilizantes tetrapirofosfato de sódio e fosfato trissódico e conservadores de } \\
\text { sorbato de potássio e nisina. }\end{array}$ \\
\hline $\mathrm{AL}$ & $\begin{array}{l}\text { Creme de leite, massa para produtos lácteos fundidos (leite pasteurizado, fermento } \\
\text { lácteo, cloreto de cálcio e coagulante), leite em pó desnatado, cloreto de sódio, } \\
\text { concentrado proteico de soro de leite, estabilizantes tetrapirofosfato de sódio e } \\
\text { fosfato trissódico e conservadores de sorbato de potássio e nisina. }\end{array}$ \\
\hline $\mathrm{BT}$ & $\begin{array}{l}\text { Creme de leite, massa para produtos lácteos fundidos (leite pasteurizado, fermento } \\
\text { lácteo, cloreto de cálcio e coagulante), leite, concentrados de proteína de leite, } \\
\text { estabilizantes pirofosfato tetrassódico, polifosfato sódico, fosfato trissódico, citrato } \\
\text { de sódio, tripolifosfato de sódio, fosfato monossódico e pirofosfato ácido de sódio, } \\
\text { cloreto de sódio e regulador de acidez. }\end{array}$ \\
\hline $\mathrm{BL}$ & $\begin{array}{l}\text { Leite, massa para produtos lácteos fundidos (leite pasteurizado, fermento lácteo, } \\
\text { cloreto de cálcio e coagulante), creme de leite, concentrado de proteína de leite, } \\
\text { estabilizantes pirofosfato tetrassódico, polifosfato de sódio, tripolifosfato de } \\
\text { potássio, pirofosfato tetrapotássico, pirofosfato ácido de sódio, fosfato trissódico, } \\
\text { citrato de sódio, tripolifosfato de sódio e fosfato monossódico, concentrado de } \\
\text { proteína de soro de leite, cloreto de sódio e regulador de acidez. }\end{array}$ \\
\hline $\mathrm{CT}$ & $\begin{array}{l}\text { Creme de leite, massa coalhada (leite pasteurizado, fermento lácteo, cloreto de cálcio } \\
\text { e coagulante), queijo mussarela, creme de soro de leite, queijo prato, concentrado } \\
\text { proteico de leite, concentrado proteico de soro de leite, soro de leite em pó, cloreto } \\
\text { de sódio, estabilizantes pirofosfato tetrassódico, polifosfato de sódio, tripolifosfato } \\
\text { de sódio e ortofosfato trissódico, regulador de acidez ácido cítrico e conservador } \\
\text { ácido sórbico. }\end{array}$ \\
\hline $\mathrm{CL}$ & $\begin{array}{l}\text { Massa coalhada (leite pasteurizado, fermento lácteo, cloreto de cálcio e coagulante), } \\
\text { creme de leite, queijo mussarela, queijo prato, creme de soro de leite, concentrado } \\
\text { protéico de soro de leite, concentrado protéico de leite, soro de leite em pó, cloreto } \\
\text { de sódio, estabilizantes pirofosfato tetrassódico, polifosfato de sódio, tripolifosfato } \\
\text { de sódio e ortofosfato trissódico, regulador de acidez ácido cítrico e conservador } \\
\text { ácido sórbico. }\end{array}$ \\
\hline $\mathrm{DT}$ & $\begin{array}{l}\text { Leite desnatado, creme de leite, água, acidulante ácido lático, estabilizante } \\
\text { polifosfato de sódio e pirofosfato de sódio, cloreto de sódio, conservantes ácido } \\
\text { sórbico e nisina. }\end{array}$ \\
\hline $\mathrm{DL}$ & $\begin{array}{l}\text { Leite desnatado, água, creme de leite, acidulante ácido lático, concentrado proteico } \\
\text { de soro de leite, estabilizantes polifosfato de sódio e pirofosfato de sódio, cloreto } \\
\text { de sódio, ácido sórbico e nisina. }\end{array}$ \\
\hline
\end{tabular}


tratamento prévio de 2 minutos em $150{ }^{\circ} \mathrm{C}$ são mais susceptíveis à desnaturação do que amostras que permanecem o mesmo tempo em $130{ }^{\circ} \mathrm{C}$ ou $100{ }^{\circ} \mathrm{C}$. Quanto maior a temperatura a qual a amostra foi exposta inicialmente, mais propícia ela esteve à desnaturação.

A respeito de $K_{\mathrm{BL}}>K_{\mathrm{BT}}$ em $25{ }^{\circ} \mathrm{C}$, a amostra BL contém estabilizantes ausentes na formulação BT. Porém, tanto tripolifosfato de potássio quanto pirofosfato tetrapotássico são termicamente estáveis, sendo improvável que tais ingredientes se comportem de maneira significativamente diferente em $10^{\circ} \mathrm{C}$ e em $50^{\circ} \mathrm{C}$. A intenção dos fabricantes pode ter sido apenas reduzir o teor de sódio da formulação, para ajustar o produto às exigências legais de redução de teor de sódio. A lista de ingredientes foi, portanto, insuficiente para prever o comportamento reológico das referidas amostras, havendo a necessidade de informações acerca das condições de processamento.

Observou-se que o aumento da taxa de cisalhamento mantém a tendência de decréscimo na viscosidade e os resultados para o produto light são inferiores àqueles do produto tradicional, conforme demonstrado nas Figuras 1, 2 e 3. Tais resultados foram condizentes com o encontrado por GonzálezTello et al. (2009), que concluiu que em taxa de cisalhamento superior a $50 \mathrm{~s}^{-1}$, o com- portamento do concentrado proteico de soro de leite apresenta comportamento newtoniano. De maneira análoga, em taxas de cisalhamento baixas houve pouca diferença entre os produtos tradicional e light, mas a viscosidade de amostras light foi mais susceptível a taxas altas de cisalhamento. Este fenômeno é demonstrado nas Figuras 1, 2 e 3. Tanto para amostras light quanto para tradicionais, os valores de $n$ não apresentaram variação consistente em função de temperatura em ambos modelos matemáticos. É particularmente interessante que o valor de $K$ para $\mathrm{BL}$ em $25^{\circ} \mathrm{C}$ é aproximadamente 20 vezes maior que o mesmo parâmetro em $10{ }^{\circ} \mathrm{C}$, enquanto o valor de $n$ é 25 vezes menor. Entretanto, a representação gráfica do modelo Herschel-Bulkley nos referidos parâmetros ( $\eta$ $\left.=(-7715) / \dot{\gamma})+7795\left(\dot{\gamma}^{0,0081-1}\right)\right)$ de fato se aproxima dos resultados obtidos. Assim, tal inconsistência parece ser devida ao perfil da curva e não a resultados equivocados.

\section{CONCLUSÃO}

As amostras de Requeijão cremoso comerciais, nas versões tradicional e light, analisadas neste estudo, atenderam a legislação específica relacionada a produtos light. Porém, das quatro amostras dessa categoria, três apresentaram um teor de umidade acima

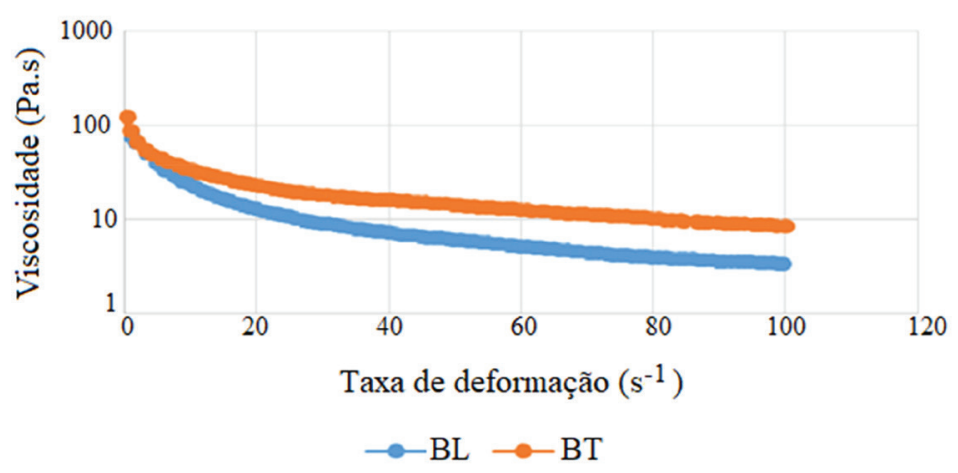

Figura 1 - Comparativo entre a viscosidade da amostra tradicional (BT) e light (BL) a $25{ }^{\circ} \mathrm{C}$ 


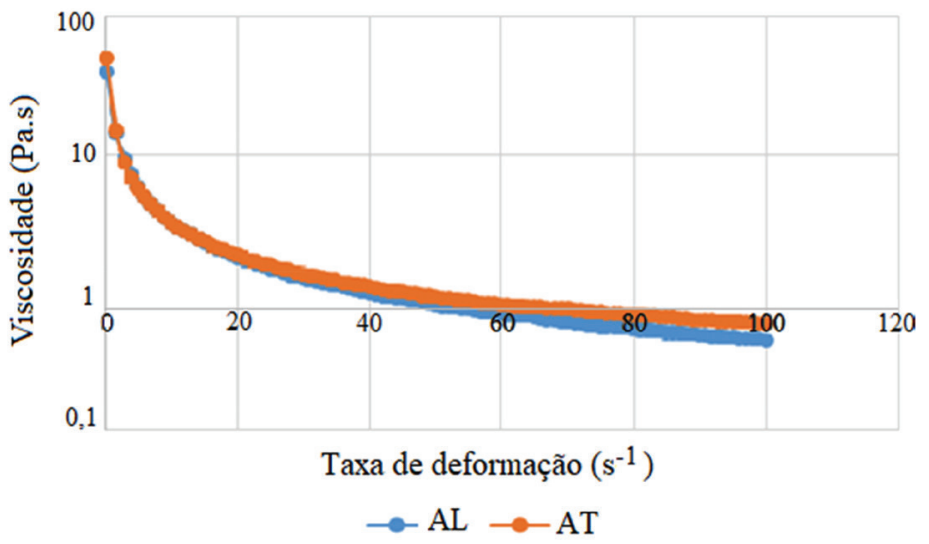

Figura 2 - Comparativo entre a viscosidade da amostra tradicional (AT) e light (AL) a $50{ }^{\circ} \mathrm{C}$

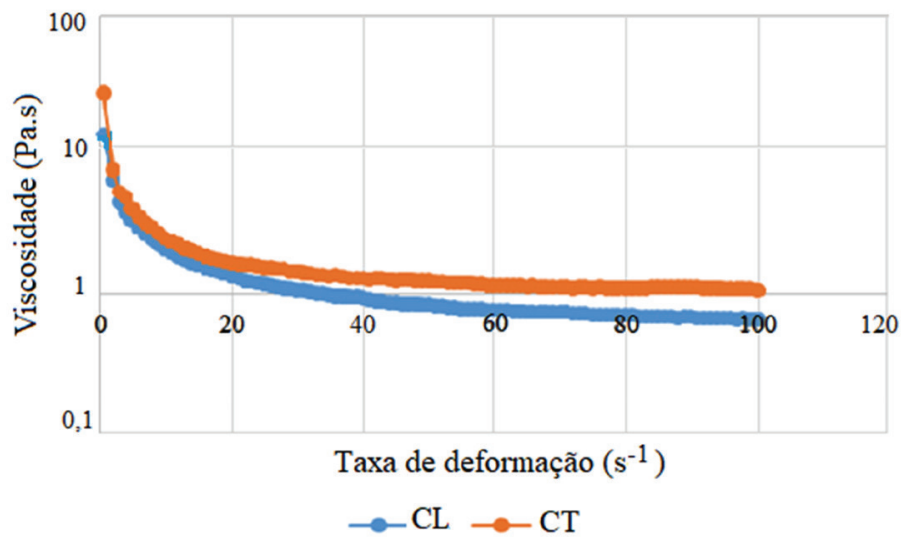

Figura 3 - Comparativo entre a viscosidade da amostra tradicional (CT) e light (CL) a $50{ }^{\circ} \mathrm{C}$

do permitido pela legislação (AL, BL, DL). Também foi possível demonstrar que duas das quatro amostras de Requeijão cremoso light apresentavam teores de proteína superiores aos teores de proteína das amostras tradicionais de mesma marca, o que é usual para obter uma viscosidade adequada do produto mesmo com a diminuição do teor de gordura. Foi possível identificar claramente o efeito da temperatura sobre as curvas de viscosidade, sendo que quanto maior a temperatura de uma dada amostra, menor os valores do índice de consistência nas condições propostas. Não foi possível identificar correlação clara entre a viscosidade e qualquer um dos itens da composição centesimal.

\section{REFERÊNCIAS}

AOAC - Association of Official Analytical Chemists. Official methods of analysis of AOAC international. $16^{\mathrm{a}}$ ed. Maryland: AOAC, 1997. 1141p.

ATUONWU, J. C.; RAY, J.; STAPLEY, A. G. F. A kinetic model for whey protein 
denaturation at different moisture contents and temperatures. International Dairy Journal, v. 75, p. 41-50, 2017.

BARONI, A. F. Caracterização reológica de requeijão cremoso tradicional e com teor reduzido de gordura: viscosidade extensional e em cisalhamento. Brazilian Journal of Food Technology, v. 2, p. 21-29, 1999.

BRASIL. Ministério da Agricultura e do Abastecimento. Portaria $n^{\circ} 359$, de 04 de setembro de 1997. Regulamento Técnico para Fixação de Identidade e Qualidade do Requeijão. Diário Oficial da República Federativa do Brasil, Brasília, 1997.

BRASIL. Ministério da Saúde. Secretaria de Vigilância Sanitária. Portaria $n^{\circ} 27$, de 13 de janeiro de 1998. Regulamento Técnico referente à Informação Nutricional Complementar. Diário Oficial da República Federativa do Brasil, Brasília, 1998.

COLLET, L. S. F. C. A.; TADINI, C. C. Sodium caseinate addition effect on the thixotropy of stirred yogurt. Anais: International Conference on Engineering and Food, ICEF 2004. p. 317-322., Montpellier, 2004.

CUNHA, C. R. Papel da gordura e do sal emulsificante em análogos de requeijão cremoso. 2007. 177 f. Tese (Doutor em Tecnologia de Alimentos) - Faculdade de Engenharia de Alimentos, Universidade Estadual de Campinas, 2007.

Dimitreli, G., THOMAREIS, A. S. Effect of chemical composition on the linear viscoelastic properties of spreadabletype processed cheese. Journal of Food Engineering, v. 84, n. 3, p. 368-374, 2008.

FU, W. et al. Effects of pre-cooked cheeses of different emulsifying conditions on mechanical properties and microstructure of processed cheese. Food Chemistry, v. 245, p. 47-52, 2018.

GARRUTI, D. S. et al. Desenvolvimento do perfil sensorial e aceitação de requeijão cremoso. Ciência e Tecnologia de Alimentos, v. 23, n. 3, p. 434-440, 2003.

GONZÁlez TELLO, P. et al. Density, viscosity and surface tension of whey protein concentrate solutions. Journal of Food Process Engineering, v. 32, n. 2, p. 235-247, 2009.

INSTituto ADOLFO LUTZ. Métodos físico-químicos para análise de alimentos. $4^{\mathrm{a}}$ ed., $1^{\mathrm{a}}$ ed. digital. São Paulo: Instituto Adolfo Lutz, 2008. 1018 p.

LÜBECK, G. M. Estudo da fabricação de requeijão cremoso com diferentes concentrações de gordura no extrato seco, sal emulsificante e concentrado proteico de soro obtido por ultrafiltração. 2005. $291 \mathrm{f}$. Tese (Doutorado em Tecnologia de Alimentos) - Faculdade de Engenharia de Alimentos, Universidade Estadual de Campinas, 2005.

MATHIAS, T. R. S. et al. Rheological evaluation of different commercial yoghurts. Brazilian Journal of Food Technology, v. 16, n. 1, p. 12-20, 2013.

MILKPOINT. As grandes oportunidades do mercado de queijos no Brasil. 2015. Disponível em: <https://www.milkpoint.com. br/noticias-e-mercado/panorama-mercado/ as-grandes-oportunidades-do-mercado-dequeijos-no-brasil-93301n.aspx>. Acesso: fev. 2018.

OLIVEIRA, T. P. et al., Avaliação físicoquímica de diferentes marcas de requeijão cremoso. In: CONGRESSO ESTADUAL 
DE MEDICINA VETERINÁRIA, 18, 2016, Canela. Anais... Canela: UFPEL, v. 4, n. 1 SE, 2016.

PASEEPHOL, T.; SMALL, D. M.; SHERKAT, F. Rheology and texture of set yogurt as affected by inulin addition. Journal of Texture Studies, v. 39, n. 6, p. 617-634. 2008.

RAMOS, A. M. et al. Rheological characterization of commercial sweetened condensed milk. Boletim do Centro de Pesquisa de Processamento de Alimentos, v. 32, n. 1, p. 83-92, 2014.

RAPACCI, M. Estudo comparativo das características físicas e químicas, reológicas e sensoriais do requeijão cremoso obtido por fermentação láctica e acidificação direta. Tese (Doutor em Tecnologia de Alimentos) - Faculdade de Engenharia de Alimentos,
Universidade Estadual de Campinas, 1997.

SÁ, E. M. F.; BARRETO, P. L. M.; BORDIGNON-LUIZ, M. T. Perfil reológico de queijo cremoso elaborado com soro de leite: influência de diferentes combinações de polissacarídeos. Revista do Instituto de Laticínios Cândido Tostes, v. 63, n. 363, p. 3-11, 2008.

SANTOS, P. H. et al. Influence of temperature, concentration and shear rate on the rheological behavior of malay apple (Syzygium malaccense) juice. Brazilian Journal of Food Technology, v. 19, 2016.

SILVA, F. C. Reologia do suco de acerola: efeito da concentração e da temperatura. 2000. 110 f. Dissertação (Mestrado em Engenharia de Alimentos) - Universidade Estadual de Campinas, Campinas, 2000. 